\title{
NGC 5128's Globular Cluster System: Is There a Dark Side?
}

\author{
Matthew Taylor ${ }^{1}$, Thomas Puzia ${ }^{1}$, Matias Gomez ${ }^{2}$ and \\ Kristin Woodley ${ }^{3}$ \\ ${ }^{1}$ Institute of Astrophysics, Pontificia Universidad Católica de Chile, Avenida Vicuña \\ Mackenna 4860, Santiago, Chile \\ ${ }^{1}$ email: mtaylor@astro.puc.cl; tpuzia@astro.puc.cl \\ ${ }^{2}$ Depto. de Ciencias Fisicas, Universidad Andres Bello, República 220, Santiago, Chile \\ email: matiasgomez@unab.cl \\ ${ }^{3}$ University of California, Santa Cruz, UC Observatories, 1156 High Street, Santa Cruz, USA
}

\begin{abstract}
Dynamical mass estimates $\left(\mathcal{M}_{\mathrm{dyn}}\right)$ and mass-to-light ratios $\left(\Upsilon_{\mathrm{dyn}}\right)$ were derived for a sample of NGC 5128's globular clusters (GCs). We find two distinct sequences in the $\Upsilon_{\mathrm{dyn}}-\mathcal{M}_{\mathrm{dyn}}$ plane, which are well fit by power laws of the forms $\Upsilon_{\mathrm{dyn}} \propto \mathcal{M}_{\mathrm{dyn}}^{0.33 \pm 0.04}$ and $\Upsilon_{\mathrm{dyn}} \propto \mathcal{M}_{\mathrm{dyn}}^{0.91 \pm 0.04}$. The former traces bright "classical" GCs, and the latter represents objects that are dynamically dominated by large dark matter components or by abnormally massive central black holes.
\end{abstract}

Keywords. galaxies:individual:NGC 5128; galaxies:globular clusters:general

\section{Introduction}

Large globular cluster (GC) systems commonly found around giant galaxies are useful tracers of the formation histories of their hosts (Brodie \& Strader 2006). GCs populate a narrow plane defined by their structural parameters (Djorgovski 1995) and are accepted to lack dark matter (DM), but this may not be theoretically expected (e.g. Peebles 1984).

\section{Conclusions}

NGC 5128 seems to have two types of high- $\mathcal{M}_{\text {dyn }}\left(\gtrsim 10^{6} M_{\odot}\right)$ GCs that follow distinct power-laws in the $\Upsilon_{\mathrm{dyn}}-\mathcal{M}_{\mathrm{dyn}}$ plane. One population is simply the bright tail of the GC luminosity function, i.e. bright but otherwise "classical" GCs. The other group is made up of objects with significant, concentrated DM components and/or they host massive central BHs that dominate the cluster dynamics out to large radii. Other artificial effects were ruled out, so we suggest that the histories of these "high- $\Upsilon_{\text {dyn }}$ " clusters are markedly different from "classical" GCs, with potentially important cosmological implications.

\section{Acknowledgements}

We acknowledge the Vicerrectoría de Investigación, and Institute of Astrophysics Graduate Fund at Pontificia Universidad Católica de Chile, FONDECYT Regular Project Grant 1121005, and BASAL Center for Astrophysics and Associated Technologies PFB06.

\section{References}

Brodie, J. P. \& Strader, J., 2006, ARA\& $A$, 44, 193

Djorgovski, S., 1995, ApJ, 438, L29

Peebles, P. J. E.., 1984, ApJ, 277, 470 